\title{
Erratum
}

\section{Erratum: Grimaldi et al., Loss of Tiparp Results in Aberrant Layering of the Cerebral Cortex}

In the article "Loss of Tiparp Results in Aberrant Layering of the Cerebral Cortex," by Giulia Grimaldi, Barbora Vagaska, Oleksandr levglevskyi, Elena Kondratskaya, Joel Glover, and Jason Matthews, which was published online on November 8 , 2019, an author's name appears incorrectly. Joel Glover should be Joel C. Glover. This correction does not affect the conclusions of the article. The name has been corrected in the online record.

https://doi.org/10.1523/ENEURO.0519-19.2019 